Received: $\quad 2016.03 .06$

Accepted: 2016.05.12

Published: 2017.01 .18

\title{
Up-Regulated Expression of SPRY4-IT1 Predicts Poor Prognosis in Colorectal Cancer
}

Authors' Contribution: Study Design A Data Collection B Statistical Analysis C Data Interpretation D Manuscript Preparation E Literature Search F Funds Collection G
BC 1 Wenlong Tan

D 2,3 Zi-zheng Song

EF 4 Qunfang Xu

AB 1 Xinyan Qu

D 1 Zhen Li

DE 4 Yu Wang

AG 5 Qun Yu

BG 1 Shengqi Wang
1 Department of Biotechnology, Beijing Institute of Radiation Medicine, Beijing, P.R. China

2 || Department of Medical Oncology, PLA General Hospital, Beijing, P.R. China 3 Department of Medical Oncology, Affiliated Hospital of Hebei University, Baoding, Hebei, P.R. China

4 Clinical Laboratory, Beijing Electric Power Hospital of Capital Medical University, Beijing, P.R. China

5 Department of Blood Products and Substitutes, Beijing Institute of Transfusion Medicine, Beijing, P.R. China
Corresponding Author: Source of support:
Shengqi Wang, e-mail: sqwang@bmi.ac.cn

This work was supported by a grant from the National Natural Science Foundation of China (81502682)

Background: Long non-coding RNA SPRY4 intronic transcript 1 (IncRNA SPRY4-IT1) has been reported to be associated with the progression of several cancers, but its expression level in colorectal cancer (CRC) has rarely been reported. The purpose of this study was to estimate the clinical significance of SPRY4-IT1 in CRC.

Material/Methods: The relative expression levels of SPRY4-IT1 were detected by quantitative real-time polymerase chain reaction (qRT-PCR) in diseased tissues and the adjacent normal tissues of 106 CRC patients. Chi-square method was used to evaluate the association between SPRY4-IT1 expression and the clinical features. Additionally, we assessed the overall survival at different expression levels of SPRY4-IT1 using Kaplan-Meier method. The prognostic significance of SPRY4-IT1 was estimated by Cox regression analysis.

Results: Up-regulated level of SPRY4-IT1 was detected in pathologic tissues of CRC patients compared with adjacent normal tissues $(P=0.000)$. The relative expression of SPRY4-IT1 was associated with the tumor size, the depth of invasion, lymph node invasion, distant invasion, and tumor stage $(P<0.05)$. Patients with high expression of SPRY4-IT1 had poor overall survival compared with those with high level (39.3 vs. 49.3 months, log-rank test, $P=0.016)$. Cox regression analysis showed that SPRY4-IT1 could act as an independent prognostic factor in CRC ( $\mathrm{HR}=2.341,95 \% \mathrm{Cl}=1.136-4.826, P=0.021)$.

Conclusions: $\quad$ SPRY4-IT1 might be associated with tumorigenesis and progression of CRC, and it may be a promising biomarker for prognosis in patients with CRC.

MeSH Keywords: Colorectal Neoplasms • Lynch Syndrome II • Prognosis

Full-text PDF: http://www.medscimonit.com/abstract/index/idArt/898369

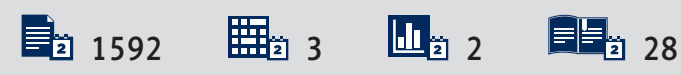




\section{Background}

Colorectal cancer (CRC) is one of the most commonly diagnosed cancers in the world, with over 600,000 deaths per year $[1,2]$. Unfortunately, the morbidity of CRC has been rapidly rising in Asian countries in recent years [3]. However, the multiple known carcinogenic factors and complex genetic backgrounds make it difficult to estimate the key factor in CRC progression [4]. At the present time, the basic prognostic biomarker in CRC is the clinicopathologic tumor staging, based on the tumor-node-metastasis (TNM) system. Nevertheless, the TNM stage is not an ideal biomarker for CRC outcomes. Patients at the same TNM stage may have different progressions and clinical outcomes due to their various genetic and epigenetic backgrounds $[5,6]$. Therefore, it is necessary to identify sensitive and specific molecular biomarkers for CRC clinical outcomes.

Long non-coding RNAs (LncRNAs) transcribed by RNA polymerase II lack open reading frames (ORF) longer than 200 nucleotides [7]. Although the functions of most IncRNAs are unknown, more and more IncRNAs are characterized and many of them are reported to regulate gene expression in the development and differentiation of diseases [8-11]. LncRNAs also have been reported to influence the development of human cancers. For example, IncRNA CCHE1 promotes cervical cancer cell proliferation [12], HNF1A-AS1 regulates proliferation and metastasis in lung adenocarcinoma [13], and MALAT1 is associated with poor prognosis of glioma [14]. SPRY4-IT1 is significantly increased in plasma samples of NSCLC patients and can act as a biomarker in NSCLC [15]. TRPM2-AS can act as a novel biomarker and therapeutic target in prostate cancer [16]. In this study, we focused on IncRNA SPRY4 intronic transcript 1 (SPRY4-IT1), which is located within an intron of the SPRY4 gene. SPRY4-IT1 was previously reported to be up-regulated in melanoma, gastric cancer, breast cancer, and esophageal squamous cell carcinoma [17-20]. However, the effect of SPRY4-IT1 in CRC prognosis is unknown.

In the present study, we applied different methods in analyzing the association between SPRY4-IT1 expression and clinical features, aiming to determine the clinical influences of SPRY4IT1 in CRC patients and to discover a reliable predictor for CRC.

\section{Material and Methods}

\section{Patients and clinical features collection}

In this study, 106 CRC patients confirmed by pathological and clinical diagnoses at the PLA General Hospital were enrolled from October 2008 to January 2014. This study was approved by the Ethics Committee of PLA General Hospital, and written consent was obtained from all the patients. Tumor and adjacent normal tissues were obtained from the CRC patients before they received any chemotherapy or radiotherapy. All the tissue samples were stored in liquid nitrogen until they were utilized.

In order to observe the results of the surgery, follow-up was performed every 3 months in the first 2 years and then every 6 months until the end of the study. All the patients were enrolled in the surgery. Overall survival was used to estimate the influence of SPRY4-IT1 on CRC patient prognosis.

\section{RNA extraction}

Total RNA was extracted from all the tissues using TRlzol reagent according to the manufacturer's instructions. The extracted RNA was dissolved in diethyl pyrocarbonate (DEPC)-water and then treated by DNase to remove DNA. The concentration of the total RNA was detected by UV absorbance at 260 $\mathrm{nm}$ and $280 \mathrm{~nm}$ (A260/A280). We used 1\% agarose gel electrophoresis to check the quality of the total RNA.

\section{Fluorescence quantitative real-time PCR}

Fluorescence quantitative real-time PCR (qRT-PCR) was used to assess the relative expression levels of SPRY4-IT1 in pathologic and adjacent normal tissues of CRC patients. The complementary DNA (CDNA) temples enrolling in the qRT-PCR were from the PrimeScript RT reagent kit (Takara, China). The qRT-PCR was performed with SYBR Green assay (Takara, China). The expression of glyceraldehyde-3-phosphate dehydrogenase (GAPDH) was used for normalized control. The data were analyzed by $2^{-\Delta \Delta c t}$ method. The primers sequences are shown in Table 1.

Table 1. The sequences of primers used in this study.

\begin{tabular}{|c|c|c|}
\hline Name & & Sequences \\
\hline \multirow{2}{*}{ SPRY4-IT1 } & Forward & 5'-ATCCGAAGCGCAGACACAATTCA- $3^{\prime}$ \\
\hline & Reverse & 5'-CCTCGATGTAGTCTATGTCATAGGA-3' \\
\hline \multirow{2}{*}{ GAPDH } & Forward & 5'-AGACTCGCTGATGATCCATGC-3' \\
\hline & Reverse & 5'-AGGTGACCACAGTGTTCTG-3' \\
\hline
\end{tabular}




\section{Statistical analysis}

Statistical analysis was completed in SPSS 18.0 software. Student's t-test was used to estimate the different expression levels of SPRY4-IT1 and the data are shown as mean \pm standard deviation (SD). The association between the clinical features and SPRY4-IT expression was evaluated by chi-square method. Kaplan-Meier method with log-rank test was applied to analyze the overall survival of the CRC patients, and univariate and multivariate Cox regression analysis were used to evaluate the prognostic value of SPRY4-IT1. $P<0.05$ was considered statistical significance.

\section{Results}

\section{Different expression of SPRY4-IT1 in CRC tissues and normal tissues}

The 106 CRC patients enrolled in this study included 52 men and 54 women with an average age of 55.02 years old. The clinical data of the participators are summarized in Table 2. QRTPCR was used to evaluate the relative expression of SPRY4IT1 in CRC tissues and normal tissues. The results indicated that the relative expression of SPRY4-IT1 in pathologic tissues was significantly higher than that in the adjacent normal tissues $(P=0.000$, Figure 1$)$.

\section{Relationship between SPRY4-IT1 expression and clinical characteristics}

To evaluate the association between SPRY4-IT1 expression and clinical features, the CRC patients were divided into high and low expression groups on the basis of their average expression of SPRY4-IT1. The chi-square results are shown in Table 2, which shows that the expression levels of SPRY4-IT1 were associated with the tumor size $(P=0.013)$, the depth of invasion $(P=0.004)$, lymph node metastasis $(P=0.017)$, distant invasion $(P=0.012)$, and tumor stage $(P=0.015)$. However, there was not significant relationship between the expression levels and sex, age, tumor location, histological differentiation, venous invasion, or nerve invasion ( $P>0.05)$. The results suggest that the expression level of SPRY4-IT1 might be associated with the development of the CRC.

\section{Overall survival analysis}

Overall survival analysis was conducted by Kaplan-Meier method, showing that the CRC patients with high expression of SPRY4-IT1 had low overall survival (the average overall survival was 39.3 months), while the low expression patients had an average overall survival of 49.3 months (Figure 2). In other words, differences between the 2 groups were significant (log-rank test, $P=0.016$ ).

\section{Univariate and multivariate Cox regression analysis for CRC prognosis}

In this study, we used Cox regression analysis to estimate the prognostic value of SPRY4-IT1. The results of univariate analysis show that the levels of SPRY4-IT1 were significantly associated with the poor prognosis in CRC patients $(P=0.021)$. Multivariate analysis suggests that SPRY4-IT1 is an independent factor for CRC prognosis ( $\mathrm{HR}=2.341,95 \% \mathrm{Cl}=1.136-4.826$, $P=0.021)$. The results of univariate and multivariate Cox regression analysis are summarized in Table 3.

\section{Discussion}

Despite great progress in early diagnosis, surgical techniques, and chemotherapy, the prognosis of patients with CRC is still unsatisfactory [21]. Prognostic factors are helpful in determining the therapeutic regimen in cancers. Due to the complex genetic background and epigenetic factors, there is still no highly sensitive and specific biomarker for CRC clinical outcomes, so the prognosis of CRC patients is poor [4]. Therefore, finding reliable biomarkers may improve the treatment of CRC.

Recently, many IncRNAs have been reported to play significant regulatory roles in human diseases [22]. Cancer-specific IncRNAs have been proven to contribute in tumor progression and serve as prognostic factors in many types of cancer. Wu et al. reported that InCRNA UCA1 could be a promising biomarker for early detection and prognosis in gastric cancer [23]. LncRNA $B A N C R$ was proved to regulate the growth and metastasis of the retinoblastoma cells and act as a prognostic target [24]. Chen et al. demonstrated that IncRNA HOTTIP promoted pancreatic cancer cell proliferation, survival, and migration [25]. As a result, the association between CRC and IncRNAs may also provide significant information about the development of cancer and clinical outcomes.

LnCRNA SPRY4-IT1 is a novel InCRNA, which was first reported to be associated with molecular etiology in human melanoma [26]. It is a 687nt unspliced, polyadenylated transcript, localized at chromosome 5q31.3. In this study, we detected the relative expression levels of SPRY4-IT1 in CRC patients using qRT-PCR (GAPDH as normalized control), aiming to estimate the clinical significance of SPRY4-IT1 in CRC. The results showed that the relative expression of SPRY4-IT1 was much higher in diseased tissues than in the corresponding adjacent normal tissues and there were significant differences between them. The abnormal expression of SPRY4-IT1 might be associated with CRC progression. We also analyzed the relationship between the SPRY4-IT1 expression and the clinical characteristics by chi-square method. The results indicated that the relative expression levels of SPRY4-IT1 were related to 
Table 2. The clinical features of the CRC patients in this study.

\begin{tabular}{|c|c|c|c|c|c|}
\hline \multirow{2}{*}{ Characteristics } & \multirow{2}{*}{$\begin{array}{l}\text { Total number } \\
\text { (n) }\end{array}$} & \multicolumn{2}{|c|}{ SPRY4-IT 1 expression } & \multirow{2}{*}{$\chi^{2}$} & \multirow{2}{*}{$\boldsymbol{P}$} \\
\hline & & High (n) & Low $(n)$ & & \\
\hline Gender & & & & 0.503 & 0.478 \\
\hline Men & 57 & 33 & 24 & & \\
\hline Women & 59 & 25 & 24 & & \\
\hline Age & & & & 0.022 & 0.882 \\
\hline$\geq 55$ & 61 & 33 & 28 & & \\
\hline$<55$ & 45 & 25 & 20 & & \\
\hline Tumor size & & & & 6.177 & 0013 \\
\hline$\geq 5 \mathrm{~cm}$ & 66 & 37 & 19 & & \\
\hline$<5 \mathrm{~cm}$ & 50 & 21 & 29 & & \\
\hline Location & & & & 0.412 & 0.521 \\
\hline Colon & 60 & 29 & 21 & & \\
\hline Rectum & 56 & 29 & 27 & & \\
\hline Histological differentiation & & & & 1.211 & 0.271 \\
\hline Well & 67 & 34 & 23 & & \\
\hline Poor & 49 & 24 & 25 & & \\
\hline The depth of invasion & & & & 8.183 & 0.004 \\
\hline \multicolumn{6}{|l|}{$\mathrm{T} 1+\mathrm{T} 2$} \\
\hline \multicolumn{6}{|l|}{$\mathrm{T} 3+\mathrm{T} 4$} \\
\hline Lymph node metastasis & & & & 5.665 & 0.017 \\
\hline Absent & 55 & 24 & 31 & & \\
\hline Present & 51 & 34 & 17 & & \\
\hline Venous invasion & & & & 0.101 & 0.751 \\
\hline Absent & 57 & 32 & 25 & & \\
\hline Present & 49 & 26 & 23 & & \\
\hline Nervous invasion & & & & 0.212 & 0.645 \\
\hline Absent & 60 & 34 & 26 & & \\
\hline Present & 56 & 24 & 22 & & \\
\hline Distant invasion & & & & 6.344 & 0.012 \\
\hline Absent & 52 & 22 & 30 & & \\
\hline Present & 54 & 36 & 18 & & \\
\hline Tumor stage & & & & 6.177 & 0.015 \\
\hline $1+11$ & 57 & 25 & 32 & & \\
\hline III+IV & 49 & 33 & 16 & & \\
\hline
\end{tabular}




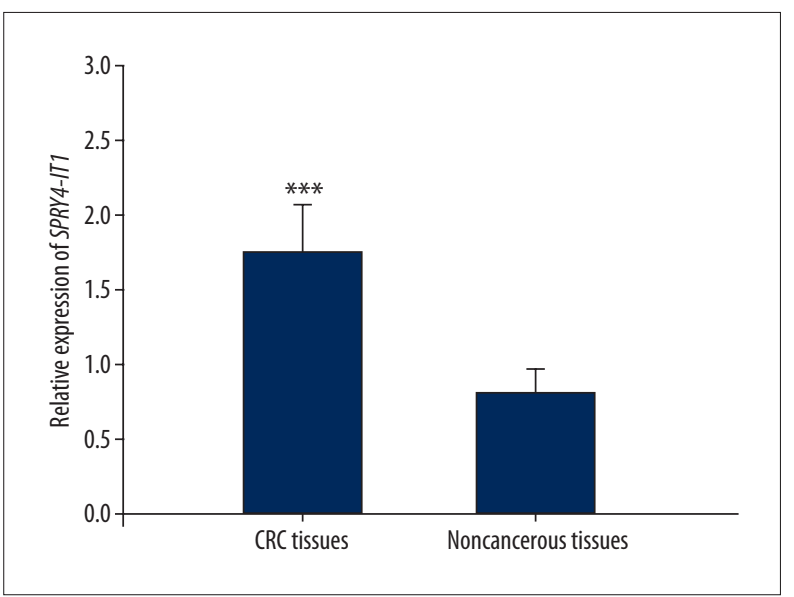

Figure 1. Relative expression of SPRY4-IT1 in CRC patients. Up-regulated level of SPRY4-IT1 was detected in CRC tissues compared with adjacent normal tissues (GAPDH as normalized control).* Indicated $P<0.001$.

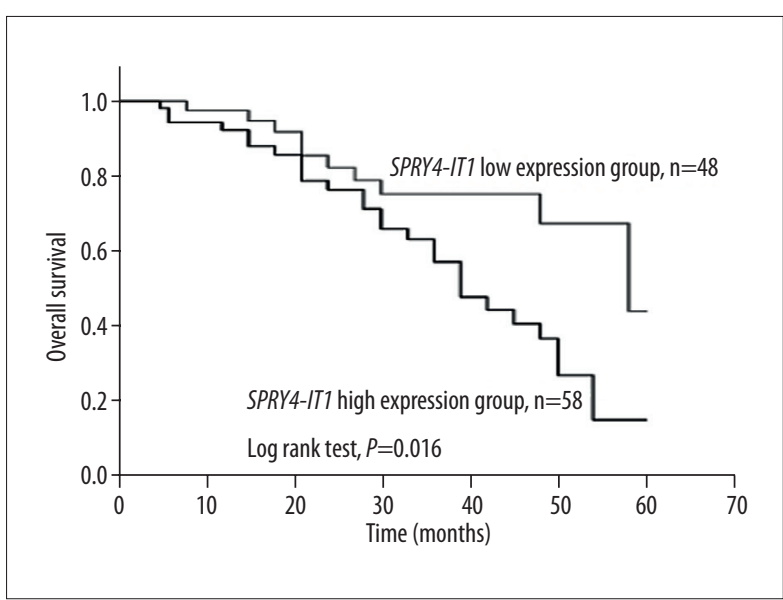

Figure 2. Overall survival analysis for patients with CRC. Patients with low level of SPRY4-IT1 had better outcomes compared with those with high level (log-rank test, $P=0.016)$.

Table 3. Cox regression analysis for prognosis in CRC patients.

\begin{tabular}{|c|c|c|c|c|c|c|}
\hline \multirow{2}{*}{ Characteristics } & \multicolumn{3}{|c|}{ Univariate analysis } & \multicolumn{3}{|c|}{ Multivariate analysis } \\
\hline & HR & $95 \% \mathrm{CI}$ & $\boldsymbol{P}$ & HR & $95 \% \mathrm{Cl}$ & $\boldsymbol{P}$ \\
\hline SPRY4-IT1 & 2.341 & $1.136-4.826$ & 0.021 & 2.341 & $1.136-4.826$ & 0.021 \\
\hline Gender & 1.297 & $0.677-2.487$ & 0.433 & - & - & - \\
\hline Age & 0.897 & $0.470-1.713$ & 0.742 & - & - & - \\
\hline Tumor size & 1.299 & $0.684-2.467$ & 0.423 & - & - & - \\
\hline Location & 0.986 & $0.521-1.866$ & 0.966 & - & - & - \\
\hline Histological differentiation & 1.214 & $0.637-2.314$ & 0.556 & - & - & - \\
\hline The depth of invasion & 0.816 & $0.425-1.566$ & 0.540 & - & - & - \\
\hline Lymph node invasion & 0.949 & $0.500-1.801$ & 0.872 & - & - & - \\
\hline Venous invasion & 0.941 & $0.493-1.795$ & 0.853 & - & - & - \\
\hline Nervous invasion & 0.826 & $0.435-1.571$ & 0.561 & - & - & - \\
\hline Distant invasion & 1.236 & $0.648-2.357$ & 0.521 & - & - & - \\
\hline Tumor stage & 0.825 & $0.433-1.571$ & 0.557 & - & - & - \\
\hline
\end{tabular}

'-' - Indicated no available data.

the tumor size, the depth of invasion, lymph node invasion, distant invasion, and tumor stage. SPRY4-IT1 expression was not relevant for sex, age, tumor location, histological differentiation, venous invasion, or nervous invasion. These data suggest that SPRY4-IT1 may play a role in the tumorigenesis and progression in CRC.

The prognostic value of SPRY4-IT1 in CRC was also analyzed in this study. Over-expression of SPRY4-IT1 was correlated with low overall survival and the Cox regression analysis showed that SPRY4-IT1 could be an independent prognostic biomarker for CRC. Similar results were also found in other types of cancer, and higher expression of SPRY4-IT1 predicted poor prognosis in many cancers, such as gastric cancer [18], esophageal squamous cell carcinoma [20], clear cell renal cell carcinoma [27], and non-small cell lung cancer [28]. These studies indicated that SPRY4-IT1 might be useful for providing insights into mechanisms of cancers development. 


\section{Conclusions}

This study proves that SPRY4-IT1 expresses aberrantly in diseased tissues of CRC patients compared with the adjacent normal tissues. Moreover, the expression level is associated with tumor size, the depth of invasion, lymph node invasion,

\section{References:}

1. Parkin DM, Bray F, Ferlay J, Pisani P: Global cancer statistics, 2002. Cancer J Clin, 2005; 55: 74-108

2. Siegel R, Naishadham D, Jemal A: Cancer statistics, 2013. Cancer J Clin, 2013; 63: 11-30

3. Sung JJ, Ng SC, Chan FK et al: An updated Asia Pacific Consensus Recommendations on colorectal cancer screening. Gut, 2015; 64: 121-32

4. Han Y, Yang YN, Yuan HH et al: UCA1, a long non-coding RNA up-regulated in colorectal cancer influences cell proliferation, apoptosis and cell cycle distribution. Pathology, 2014; 46: 396-401

5. Dong Y, Yu J, Ng SS: MicroRNA dysregulation as a prognostic biomarker in colorectal cancer. Cancer Manag Res, 2014; 6: 405-22

6. Reimers MS, Zeestraten EC, Kuppen PJ et al: Biomarkers in precision therapy in colorectal cancer. Gastroenterol Rep (Oxf), 2013; 1: 166-83

7. Kornienko AE, Guenzl PM, Barlow DP, Pauler FM: Gene regulation by the act of long non-coding RNA transcription. BMC Biol, 2013; 11: 59

8. Ponting CP, Oliver PL, Reik W: Evolution and functions of long noncoding RNAs. Cell, 2009; 136: 629-41

9. XU XF, Li J, Cao YX et al: Differential expression of long noncoding RNAs in human cumulus cells related to embryo developmental potential: A microarray analysis. Reprod Sci, 2015; 22: 672-78

10. Chalei V, Sansom SN, Kong L et al: The long non-coding RNA Dali is an epigenetic regulator of neural differentiation. Elife, 2014; 3: e04530

11. Taft RJ, Pang KC, Mercer TR et al: Non-coding RNAs: regulators of disease. J Pathol, 2010; 220: 126-39

12. Yang $M$, Zhai $X$, Xia $B$ et al: Long noncoding RNA CCHE1 promotes cervical cancer cell proliferation via upregulating PCNA. Tumour Biol, 2015; 36 7615-22

13. Wu Y, Liu $H$, Shi $X$ et al: The long non-coding RNA HNF1A-AS1 regulates proliferation and metastasis in lung adenocarcinoma. Oncotarget, 2015; 6: 9160-72

14. Ma KX, Wang HJ, Li XR et al: Long noncoding RNA MALAT1 associates with the malignant status and poor prognosis in glioma. Tumour Biol, 2015; 36: 3355-59

15. Hu X, Bao J, Wang Z et al: The plasma IncRNA acting as fingerprint in nonsmall-cell lung cancer. Tumour Biol, 2016; 37: 3497-504 distant invasion, and tumor stage. Additional, SPRY4-IT1 can act as an independent biomarker for CRC prognosis and overexpression of SPRY4-IT1 predicts poor prognosis in CRC. These results suggest that SPRY4-IT1 may be a promising target for CRC therapy.

16. Mouraviev V, Lee B, Patel V et al: Clinical prospects of long noncoding RNA as novel biomarkers and therapeutic targets in prostate cancer. Prostate Cancer Prostatic Dis, 2016; 19: 14-20

17. Mazar J, Zhao W, Khalil AM et al: The functional characterization of long noncoding RNA SPRY4-IT1 in human melanoma cells. Oncotarget, 2014; 5 8959-69

18. Peng W, Wu G, Fan $\mathrm{H}$ et al: Long noncoding RNA SPRY4-IT1 predicts poor patient prognosis and promotes tumorigenesis in gastric cancer. Tumour Biol, 2015; 36: 6751-58

19. Shi Y, Li J, Liu Y et al: The long noncoding RNA SPRY4-IT1 increases the proliferation of human breast cancer cells by upregulating ZNF703 expression. Mol Cancer, 2015; 14: 51

20. Xie HW, Wu QQ, Zhu B et al: Long noncoding RNA SPRY4-IT1 is upregulated in esophageal squamous cell carcinoma and associated with poor prog nosis. Tumour Biol, 2014; 35: 7743-54

21. Sung JJ, Lau JY, Goh KL, Leung WK: Increasing incidence of colorectal can cer in Asia: Implications for screening. Lancet Oncol, 2005; 6: 871-76

22. Bhan A, Mandal SS: Long noncoding RNAs: Emerging stars in gene regulation, epigenetics and human disease. Chem Med Chem, 2014; 9: 1932-56

23. Zheng $Q$, Wu F, Dai WY et al: Aberrant expression of UCA1 in gastric cancer and its clinical significance. Clin Transl Oncol, 2015; 17: 640-46

24. Su S, Gao J, Wang $T$ et al: Long non-coding RNA BANCR regulates growth and metastasis and is associated with poor prognosis in retinoblastoma. Tumour Biol, 2015; 36: 7205-11

25. Cheng Y, Jutooru I, Chadalapaka $G$ et al: The long non-coding RNA HOTTIP enhances pancreatic cancer cell proliferation, survival and migration. Oncotarget, 2015; 6: 10840-52

26. Khaitan D, Dinger ME, Mazar J et al: The melanoma-upregulated long noncoding RNA SPRY4-IT1 modulates apoptosis and invasion. Cancer Res, 2011 71: 3852-62

27. Zhang HM, Yang FQ, Yan $Y$ et al: High expression of long non-coding RNA SPRY4-IT1 predicts poor prognosis of clear cell renal cell carcinoma. Int J Clin Exp Pathol, 2014; 7: 5801-9

28. Sun M, Liu XH, Lu KH et al: EZH2-mediated epigenetic suppression of long noncoding RNA SPRY4-IT1 promotes NSCLC cell proliferation and metastasis by affecting the epithelial-mesenchymal transition. Cell Death Dis, 2014; 5: e1298 